\title{
In Silico Perspectives on the Prediction of the PLP's Epitopes involved in Multiple Sclerosis
}

\author{
Zahra Zamanzadeh ${ }^{1}$, Mitra Ataei ${ }^{1}$, Seyed Massood Nabavi², Ghasem Ahangari', Mehdi Sadeghi', \\ Mohammad Hosein Sanati ${ }^{1, *}$ \\ ${ }^{1}$ Department of medical biotechnology. Institute of Medical Genetic, National Institute of Genetics Engineering and Biotechnology \\ (NIGEB), Tehran, 14965/161 Iran \\ ${ }^{2}$ Department of Neurology, Faculty of Public Health, Shahed University, Tehran, 18155/159, Iran \\ ${ }^{*}$ Corresponding author: Department of Medical Genetic, National Institute of Genetic Engineering and Biotechnology (NIGEB), \\ Shahrak-e- Pajoohesh, 15th Km, Tehran-Karaj Highway, Tehran, 14965-161, Iran. \\ Tel.: +98 21 44780365; Fax: +98 21 44780395; E-mail: m-sanati@nigeb.ac.ir
}

Received: 15 Sep. $2015 \quad$ Revised: 29 May $2016 \quad$ Accepted: 13 March $2017 \quad$ Published online: 31 May 2017

\begin{abstract}
Background: Multiple sclerosis (MS) is the most common autoimmune disease of the central nervous system (CNS). The main cause of the MS is yet to be revealed, but the most probable theory is based on the molecular mimicry that concludes some infections in the activation of $\mathrm{T}$ cells against brain auto-antigens that initiate the disease cascade.

Objectives: The Purpose of this research is the prediction of the auto-antigen potency of the myelin proteolipid protein (PLP) in multiple sclerosis.

Materials and Methods: As there wasn't any tertiary structure of PLP available in the Protein Data Bank (PDB) and in order to characterize the structural properties of the protein, we modeled this protein using prediction servers. Meta prediction method, as a new perspective in silico, was performed to find PLPs epitopes. For this purpose, several T cell epitope prediction web servers were used to predict PLPs epitopes against Human Leukocyte Antigens (HLA). The overlap regions, as were predicted by most web servers were selected as immunogenic epitopes and were subjected to the BLASTP against microorganisms.
\end{abstract}

Results: Three common regions, $\mathrm{AA}_{58-74}, \mathrm{AA}_{161-177}$ and $\mathrm{AA}_{238-254}$ were detected as immunodominant regions through meta-prediction. Investigating peptides with more than $50 \%$ similarity to that of candidate epitope $\mathrm{AA}_{58-74}$ in bacteria showed a similar peptide in bacteria (mainly consistent with that of clostridium and mycobacterium) and spike protein of Alphacoronavirus 1, Canine coronavirus, and Feline coronavirus. These results suggest that cross reaction of the immune system to PLP may have originated from a bacteria or viral infection, and therefore molecular mimicry might have an important role in the progression of MS.

Conclusions: Through reliable and accurate prediction of the consensus epitopes, it is not necessary to synthesize all PLP fragments and examine their immunogenicity experimentally (in vitro). In this study, the best encephalitogenic antigens were predicted based on bioinformatics tools that may provide reliable results for researches in a shorter time and at a lower cost.

Keywords: Epitope Prediction; Human Leukocyte Antigens; Multiple Sclerosis; Molecular Mimicry; Myelin Proteolipid Protein (PLP)

\section{Background}

As the most common autoimmune disease of the central nervous system (CNS), multiple sclerosis (MS) is a chronic inflammatory demyelinating health problem of the CNS which leads to the neurological disability in young adults (1-3). In MS, T cells attack the myelin sheath of the neurons in the CNS, destroying the myelin and the axons (4). The Etiology of MS is unknown, but the most probable theory is based on the molecular mimicry that establishes a number of infections which can activate T cells and initiate the disease's cascade. In this model, induced auto-aggressive $\mathrm{T}$ cells are followed by structural similarity between microbial epitopes and self-peptides. Therefore, Molecular mimicry can break self-tolerance and induce autoimmune responses. In $\mathrm{T}$ cell mediated auto-immunities, transfer of activated $\mathrm{T}$ 
cell to the animal models can lead to autoimmunity. It is noteworthy that there are some auto-antigens in the brain including Myelin Basic Protein (MBP), proteolipid protein (PLP), myelin oligodendrocyte glycoprotein (MOG), myelin associated glycoprotein (MAG), alpha-beta crystalline, and heat shock proteins that induce $\mathrm{T}$ cell reactivity and play a crucial role in commencing MS (5-10).

One of the brain's auto-antigens is proteolipid protein (PLP); the most abundant autoantigen in the CNS myelin. It plays an important role in the stability of the structure and the function of the myelinated neurons $(11,12)$. Solely, PLP composes about $50 \%$ of the total CNS myelin proteins. The protein has been isolated by Folch and Lees in $1951(13,14)$. PLP is able to induce autoimmune encephalomyelitis experimentally in the rodents and non-human primates. Its post-translational modification, acylation, makes PLP an effective autoantigen $(11,15)$.

PLP consists of 276 amino acid residues and four highly hydrophobic trans- membrane domains. In adults, PLP expression is limited to the oligodendrocyte cells (16).

PLP is a protein with a highly conserved sequence. The amino acid sequence of the PLP protein from bovine, rat, mouse, dog, and human are $99 \%$ identical, suggesting that PLP has an imperative role in the CNS $(17,18)$. Located on chromosome 10 (Xq22.2), the 17 kbp gene of PLP protein constitutes seven exons. PLP1 encodes both PLP and its splice variant called Dm20, its 242 amino acid isoform. Dm20 mRNA lacks 105 bp of the exon 3 of the PLP mRNA; therefore, 35 amino acids of its cytosolic loop is lost (19).

Experimental autoimmune encephalomyelitis (EAE) is the animal model of MS. Based on the encephalitogenic potential in animal models and reactivity by MS T-cells, PLP epitopes were selected to induce EAE (20). Researchers have shown that PLP peptide composed of amino acids 43-64 could induce EAE in PL/J mice (21). In addition, residues 139-151, 178-191, 104-117, and 57-70 of PLP are encephalitogenic for SJL mice (22-25). Previous studies have shown that T cells can respond to the PLP40-60 (26), PLP89-106 (27), PLP3049, PLP180-199 (28), and PLP184-209 (29). Thus, if the best PLP epitopes and significant similarity between these epitopes and some microbial epitopes could be found, molecular mimicry hypothesis could be proven.

In spite of numerous experimental studies for recognition of the epitopes related to the auto-antigenic proteins of the myelin, there isn't a systemic method available for PLP epitope prediction as yet $(29,30)$. Thus far, prediction methods for the recognition of the effective PLP epitopes in MS were based on the following criteria: Reports regarding encephalitogenic potential in the animal models and preferential reactivity through MS T-cells. However, several useful bioinformatics softwares have been developed to predict the most probable epitopes.

In this study, we have employed several bioinformatics tools for T-cell epitopes prediction and similarity search helped us to choose the best epitopes which can be involved in the molecular mimicry. PLP epitopes that may cause MS by molecular mimicry mechanism can be determined with these bioinformatics tools.

\section{Objective}

The purpose of the present study was to predict the auto-antigenic immunodominant epitopes of the PLP protein and characterization of the molecular/structural characteristics of which by using in-silico approaches as a substantial step for in vitro tests.

\section{Materials and Methods}

\subsection{Sequence Retrieval, Alignment, and General Analysis}

The complete annotated sequence of the human PLP and its isoforms were obtained from the NCBI database (www.ncbi.nlm.nih.gov/). Three reference sequences were obtained and were subjected to the multiple sequence alignment by using NCBI ( (http://www.ncbi.nlm.nih.gov/tools/cobalt/cobalt. cgi?link_loc=BlastHomeLink-) in order to explore the fragments that had maximum similarity. Uniprot $\mathrm{KB}$ (www.uniprot.org/-) and NCBI databases were used to characterize more properties of PLP. Prosite (http:// prosite.expasy.org/ ) was applied to explore PLP motifs.

\subsection{Analyzing Primary and Tertiary Structure of PLP}

The primary protein structure of PLP (e.g. length, molecular weight (MW), isoelectric point (pI), amino acids, etc.) was arranged in Table 1 using Expasy tools (http://web.expasy.org/protparam/). Since we could not find any match in Protein Data Bank (PDB) for PLP to analyze its functional and structural motifs, we used phyre $^{2}$ server (http://www.sbg.bio.ic.ac.uk/ phyre2/ $\mathrm{html} /$ page.cgi?id=index) which combines learning machine methods, evolutionary information, fragment libraries and energy functions to predict protein tertiary structures and structural features. The selected model underwent energy minimization processes with the GROMOS96 method implementation of SWISS pdb Viewer software version 4 (http://www.expasy.org/spdbv/ ) and loops refined by Modeler software. The PLP 
Table 1. Parameters computed using Expasy Prot Param tool.

\begin{tabular}{ll}
\hline Accession Number (AC.) & PLP (NP_001122306.1) \\
\hline No. of amino acids & 276 \\
Mol. Wt a & 29929.9 \\
pI a & 8.71 \\
Total -R and + R a & 12,19 \\
Inst.II a & 18.92 \\
AI, GRAVY a & $91.59,0.542$ \\
\hline
\end{tabular}

a: Abbreviations; Mol. Wt, Molecular Weight; pI, Theoretical Isoelectric Point; -R, Number of negative charged residues (Arg + Lys); +R, Number of positive charged residues (Asp + Glu); EC, Extinction Coefficient at $280 \mathrm{~nm}$; II, Instability Index; AI, Aliphatic Index; GRAVY, Grand Average Hydropathicity.

model was validated with the help of Rampage server (31) and then visualized by the USCF Chimera software.

\subsection{MHC Class I and II Epitopes for T-cells}

A consensus approach (based on Artificial Neural Network (ANN), Stabilized Matrix Method (SMM) and Support Vector Machine (SVM)), was used for the prediction of MHC I and MHC II binding peptides with lengths of 8-15 amino acids. T cell epitope prediction web servers were used to predict continuous epitopes. Some of these servers are:

- HLAPred (www.imtech.res.in/raghava/hlapred/ ), which is a HLA peptide binding predictor.

- IEDB (Immune-Epitope Database) server (http:// tools.immuneepitope.org/-), which provides access to a reliable prediction of peptides binding to the MHC molecules. It estimates IC50 values for peptides binding to specific MHC molecules. Some of the most frequent alleles were used for developing $\mathrm{T}$ cell epitope prediction.

- CTLPred (http://www.imtech.res.in/raghava/ ctlpred/), which is an ANN and SVM based CTL epitope prediction tool.

- ProPred-I (http://www.imtech.res.in/raghava/ propred1/-), which predicts MHC-I binding regulation in an antigen sequence using quantitative matrices for 47 MHC-I alleles.

- ProPred (www.imtech.res.in/raghava/propred/), which predicts MHC-II binding regulation in an antigen sequence using quantitative matrices and is effective in locating promiscuous binding region used to select vaccine candidates.

- SYFPEITHI (www.syfpeithi.de), which is a predictive server featuring MHC-presented epitopes,
MHC-specific anchor, and auxiliary motifs.

Furthermore, these peptides are promiscuous in their ability to bind to a variety of MHC class I and II molecules, including those most commonly found in MS patient population (HLA-A*24:02, DRB1*15, DRB1*04, DQA1*01:02/DQB1*0602, DQA1*04:01/ DQ B $1 * 0402$, DQ A 1*05:01/DQB 1*0201, DQA $1 * 05: 01 / \mathrm{DQB} 1 * 0301)$.

B cell epitope prediction web servers were also used to predict epitopes. A number of these servers are: IEDB (http://tools.immuneepitope.org/), BCPred (http://ailab. ist.psu.edu/bcpred/predict.html ) and ABCPred (www. imtech.res.in/raghava/abcpred).

Finally, Meta prediction method was used to select the most effective epitopes. For this aim, the overlap regions which were predicted by most of the servers were selected as immunogenic regions. Web logo (http:// weblogo.berkeley.edu/logo.cgi ) was used to represent the conservation of the selected epitope sequences.

\subsection{Selected Epitopes and BLASTp}

After prediction of the consensus epitopes, three high ranked selected epitopes were directed to protein similarity BLAST search (BLASTp) (http://blast.ncbi.nlm. nih.gov/Blastp.cgi ) for the evaluation of similar peptides reported in different bacteria, viruses, and protozoa species. The selected algorithm was blastp (protein-protein BLAST), with expecting threshold of 10 , word size 3, and blosum62 matrix. Then we selected the closest hits and summarized them in an informative table.

\section{Results}

\subsection{Alignment and General Analysis}

Results obtained through NCBI database have revealed that PLP has three isoforms; isoform1 (NP_001122306.1) which is Proteolipid protein with 277 amino acid, isoform 2, DM20 (NP 955772.1) which has 35 amino acids less than PLP, and the isoform 3 (NP_001291933.1) with 222 amino acids. Their initiator methionine will be eliminated during post translation modification.

Multiple sequence alignment using NCBI showed that isoforms 2 and 3 are identical with PLP except region 117 to 152 which have been eliminated in DM20, and the region 1-55 which is lost in isoform 3 .

Uniprot KB database exhibited PLP with P60201 (MYPR_HUMAN) accession number. PLP with an alternative name of Lipophilin which is the major protein from the CNS plays a vital role in the formation or maintenance of the multilamellar structure of myelin. 
This protein is a hydrophobic integral membrane protein containing four transmembrane regions, one cytoplasmic, and two extracellular loops. The post translational modifications of the PLP are lipidation in amino acids: 6, 7, 10, 109, 139, 141, 199 and disulfide bond formation between amino acid 184, 228 and 201, 220, respectively.

Using Prosite, it was found that PLP has two motifs at positions 28-37 (PS00575) and 228-247 (PS01004) that don't have any defined function.

\subsection{PLP Protein, Primary, and the Tertiary Structures}

A summarized analysis of the data that was obtained by ExPASy ProtParam tool is presented in Table 1. Isoelectric point (pI) is important for estimating the solubility of the proteins. The calculated isoelectric point (pI) was 8.71. So, the PLP protein has a basic nature. The instability index (Inst.II) provides an estimation of the protein stability in vitro. Based on instability index, PLP could be classified as a stable protein. The high aliphatic index (91.59) shows that PLP protein is stable for a variety of temperature ranges. The Grand Average Hydropathicity (GRAVY) values showed positive results (0.542), which indicate the relatively low hydrophilicity and low interaction of the protein with the surrounding water molecules.

Physicochemical results have revealed that the most abundant amino acid residues were alanine (A) $(11 \%)$, leucine (L) (11\%), and glycine (G) (10\%). The distribution of amino acid frequency in PLP protein showed that negative R-groups are more frequent than positive R-groups.

Furthermore, as there wasn't any tertiary structure for this protein in the Protein Data Bank (PDB) in addition to the structural properties of this protein, we modeled this protein (Fig. 1). In order to achieve a higher quality of the modeled structure, energy minimization $(E=-2432.302)$ and loop refinement were done. The quality of the model was validated by Ramachandran plot. Evaluation of the quality of the model by Rampage showed that the number of residues in favor, allowed, and outlier regions were 214 (89.2\%of all residue), 22 $(9.2 \%)$, and $4(1.7 \%)$ respectively. So, the reliability of the model by Ramachandran plot was $98.4 \%$. Overall, these results revealed a valid modeled structure. Also, as seen in Figure 1, the model is composed of 13 coil regions (Coil), 11 helices $(\mathrm{H})$, and 4 strands or beta sheet (E) substructures. A higher rate of the residue with the helix substructure reflects the transmembrane nature of the protein. As we analyzed later in the MHC prediction binding peptides, most of the epitope located in the helix regions and coil stretches may have roles in stimulation of the humoral immune response (besides

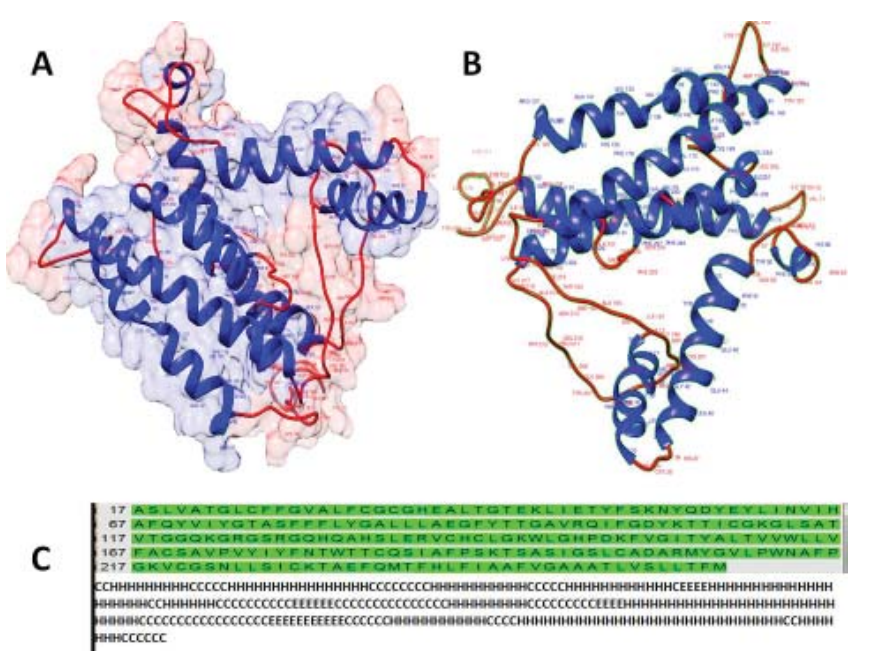

Figure 1. Visualizations of the predicted tertiary A: ribbon presentation with surface transparency $(90 \%) \&$ B: ribbon representation and secondary structure of the PLP protein (C) by Chimera software 1.8 .

cellular immune response) by antibodies.

4.2. MHCI \& MHC II Binder Peptides (T cell Epitopes) Applying the consensus approach results obtained for PLP interactions with the T-Cell epitopes (MHC I \& MHC II binder peptides) are arranged in Table 2. This table includes peptides with higher affinities (low ranks) for the corresponding alleles while peptides with lower affinities were ignored.

\subsection{Selecting Epitopes for In vitro Testing}

Finally, three common regions were selected as immunogenic (i.e., immunodominant) epitopes for stimulation of the both MHCI \& MHCII alleles based on the predicted peptides with higher ranks for in vitro testing. These results were visualized as shown in Figure 2. These regions are included of the following peptides: the $\mathrm{AA}_{58-74}$ YEYLINVIHAFQYVIYG peptide sequence, the $\mathrm{AA}_{161-177}$ region: composed of VVWLLVFACSAVPVYIY amino acids, and the last region; $\mathrm{AA}_{238-254}$ which was composed of the peptide with subsequent amino acids HLFIAAFVGAAATLVSL. Web logo was used to evaluate the consensus sequence of the three selected zones and it was shown that these three regions are conserved sequences (Fig. 3).

The antigenic criteria proposed for the selection of these sequences were:

- The epitope region is recognized by the minimum number of fragments and the maximum properties for selection of an epitope function 
Table 2. Predicted MHC I \& MHC II binder peptides for HLA-A*24:02, HLA-DRB1*15 (subtypes), HLA-HLADRB1*04:01, HLA-DQA1*05:01/DQB1*03:01, HLA-DQA1*04:01/DQB1*04:02 and DQA1*01:02/DQB1*06:02. Results were arranged in their corresponding boxes based on peptides with higher scores for each allele. Arranged based on their rank. Low percentile rank $=$ good binders.

\begin{tabular}{|c|c|c|}
\hline HLA type & Tools & Positions \\
\hline HLA-A24 & BIMAS & $262-270,156-165,72-81,156-164$ \\
\hline HLA-A24 & HLAPred & $81-89,130-138,37-45,210-218$ \\
\hline HLA-A24 & IEDB & $156-167,176-187$ \\
\hline HLA-A24 & NetMHC & $\begin{array}{l}71-79,231-239,255-263,155-163,71-80,205-213,155-165,175-186,155-166,49-61 \\
48-61,173-186,175-188\end{array}$ \\
\hline HLA-A24 & ProPred-I & $262-170,156-163$ \\
\hline HLA-A24 & SYFPEITHI & $262-270,156-165,54-62,59-68$ \\
\hline HLA-A24 & $\begin{array}{l}156-164,262-270 \\
\text { CTLPred }\end{array}$ & \\
\hline HLA-DRB $1 * 15$ & HLAPred & $79-87,70-78,174-182,251-259$ \\
\hline HLA-DRB $1 * 15$ & IEDB & $74-88,76-90,249-263,245-259$ \\
\hline HLA-DRB $1 * 15$ & ProPred & $78-86,173-181,250-258,69-77,163-171$ \\
\hline HLA-DRB $1 * 15$ & SYFPEITHI & $206-220,76-90,169-183$ \\
\hline HLADRB $1 * 0401$ & HLAPred & $79-87,70-78,177-185,58-66,245-53,251-259,161-169$ \\
\hline HLADRB $1 * 0401$ & IEDB & $\begin{array}{l}251-265,252-266,253-267,254-268,255-269,256-270,257-271,239-253,241-255 \\
242-256,240-255,243-257,249-263,250-263,246-260,247-261,248-262,55-69,54- \\
68,53-67,173-187,174-188,175-189,172-186,171-185,56-70,52-66,66-80,65-79, \\
67-81,64-78,68-82\end{array}$ \\
\hline HLADRB $1 * 0401$ & ProPred & $\begin{array}{l}176-184,57-65,244-252,177-185,250-258,256-264,171-178,69-77,160-168,174- \\
182\end{array}$ \\
\hline HLADRB $1 * 0401$ & SYFPEITHI & $172-186$ \\
\hline DQA $1 * 01: 02 / D Q B 1 * 0602$ & IEDB & $\begin{array}{l}241-255,242-256,243-257,240-254,244-258,239-253,245-259,153-167,75-89,80- \\
94,\end{array}$ \\
\hline DQA $1 * 04: 01 / \mathrm{DQB} 1 * 0402$ & IEDB & $\begin{array}{l}\text { 77-91, 78-92, 76-90, 79-93, 80-94, 68-82, 69-83, 67-81, 75-89, 70-84, 81-95, 66-80, } \\
239-253\end{array}$ \\
\hline DQA $1 * 05: 01 / \mathrm{DQB} 1 * 0201$ & IEDB & $\begin{array}{l}\text { 76-90, 77-91, 75-89, 74-88, 78-92, 73-87, 79-93, 155-169, 154-168, 239-253, 238-252, } \\
240-254,161-175,\end{array}$ \\
\hline $\mathrm{A} 1 * 05: 01 / \mathrm{DQB} 1 * 0301$ & IEDB & $\begin{array}{l}239-253,240-254,241-255,238-252,242-258,237-251,236-250,243-257,244-258 \\
235-249,234-248,75-89,76-90,77-91,78-92,74-88,9-23,10-24,8-22\end{array}$ \\
\hline
\end{tabular}

- The most different algorithms in the T cell epitope prediction databases

- Identification by B cells (linear or continuous epitope prediction)

- Epitope sequences with more conserved amino acids and the most similar sequences in different organisms
These results proposed the epitope $\mathrm{AA}_{58-74}$ as the highest priority and the most antigenic for MS to be tested in the experimental phase.

\subsection{BLAST of Predicted Epitopes}

Peptide sequences of the predicted epitopes were used as entry data to NCBI BLASTp search for finding the most similar peptides in bacteria, viruses, and protozoa 


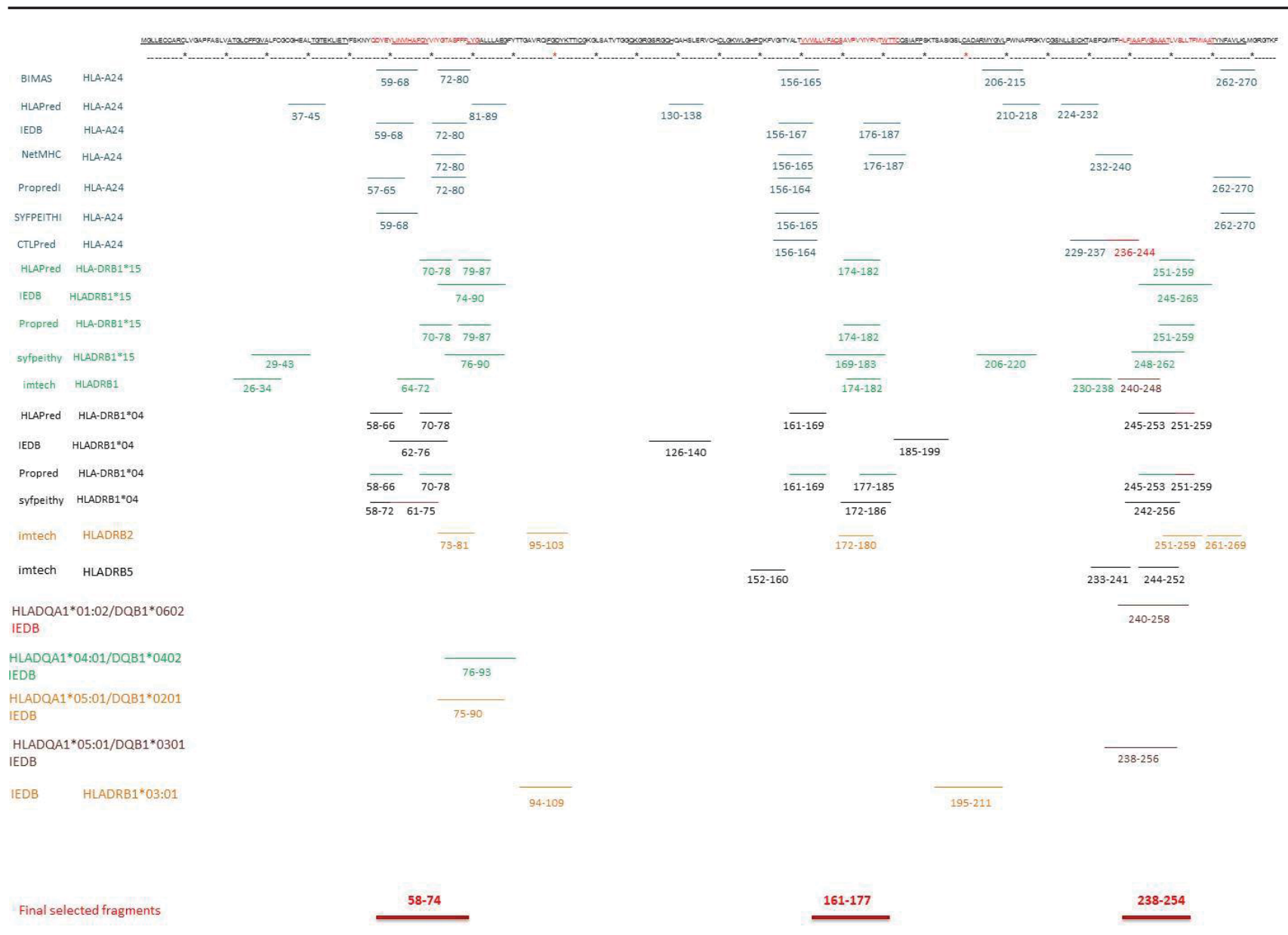

Figure 2. Graphical presentation of the predicted epitopes on PLP protein amino acid sequence. Three selected epitopes $\left(\mathrm{AA}_{58-74}, \mathrm{AA}_{161-177}\right.$, and $\left.\mathrm{AA}_{238-254}\right)$ were predicted to be immunogenic regions as indicated by most database sites.

species. BLAST results for the three candidate epitopes and protozoa with $>50 \%$ similarity didn't show any significant similarity; therefore, protozoa couldn't induce immune cell reactivity to these epitopes of myelin PLP.

BLAST was carried out for the candidate epitope $\mathrm{AA}_{58-74}$ (YEYLINVIHAFQYVIYG) and bacteria with $>50 \%$ similarity showed that the peptide fragment

Figure 3. Conserved sequences inside the three selected regions. The presence of only one amino acid in each position is an indicator for the most conserved sequence and the two or more amino acids in the position show variability.
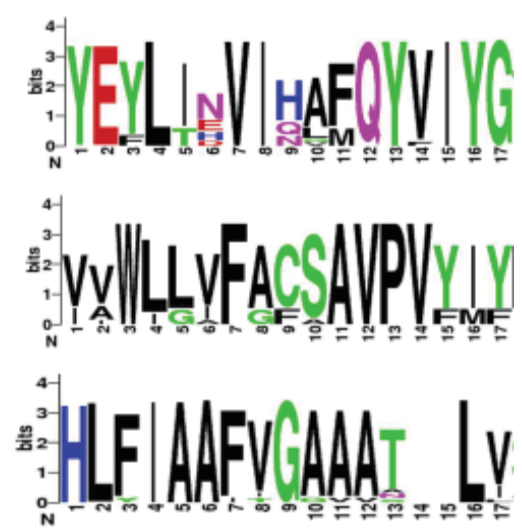

LINVIHAFQ is the most repeated region in bacteria and viruses.

\subsection{Finding Similar Epitopes in Bacteria and Viruses}

Peptide sequences of the predicted epitopes were searched in order to find similar peptides in bacteria and viruses using BLASTP. Results obtained for the peptides in the bacterial kingdom with more than $50 \%$ similarity to the candidate epitope, $\mathrm{AA}_{58-74}$ (YEYLINVIHAFQYVIYG) showed a similar peptide in the bacteria that mainly are consisted of the clostridia and mycobacteria (Table 3 ).

Investigation on the similarity between the epitope $\mathrm{AA}_{58-74}$ and viruses has led us to a region in the spike protein of the alphacoronavirus 1, Canine coronavirus, and Feline coronavirus (Table 3). Spike protein/S protein or spike glycoprotein is a major protein in the pathogens of the severe acute respiratory syndrome (SARS) associated with coronavirus (32).

These results suggest that the cross reaction of the 
Table 3. BLAST results as obtained for the peptides fragments in bacterial and viral proteins similar to the analyzed epitope, $\mathrm{AA}_{58-74}$ in amino acid sequence.

\begin{tabular}{|c|c|c|}
\hline Protein name & Sequence & Organism \\
\hline hypothetical protein & YEYLINQIIHSF & Leptospira interrogans \\
\hline histidine kinase & EFLINVIQEFEKDRQYV & Haemophilus influenza \\
\hline histidine kinase & EFLINVIQEFEKDRQYV & Eubacterium \\
\hline histidine kinase & EFLINVIQEFEKDRQYV & Peptostreptococcaceae bacterium \\
\hline histidine kinase & EFLINVIQEFEKDRQYV & Haemophilus haemolyticus \\
\hline flagellar motor protein MotP & LIDVIHAFQ & Desulfarculus sp. SPR \\
\hline predicted protein & YEYLDTVIHEFRHAMQY & Clostridiales bacterium \\
\hline $\begin{array}{l}\text { glycerol-3-phosphate } \mathrm{ABC} \\
\text { transporter permease }\end{array}$ & LINVINAFQ & Clostridium sp. LF2 \\
\hline metal dependent phosphohydrolase & EYLIKVIGAFQ & Clostridium sp. CAG:230 \\
\hline hypothetical protein & YEYLINNIH & Salmonella enterica \\
\hline $\begin{array}{l}\text { LytTr DNA-binding domain } \\
\text { protein }\end{array}$ & EYMEDVFSIHTFDYVI & Enterococcus faecalis \\
\hline hypothetical protein & FEYLIKVIPAF & Clostridium asparagiforme \\
\hline Wzy & YQYLINGIRAF & Cronobacter muytjensii \\
\hline RND transporter & YDYLINVLH & Acinetobacter johnsonii, A. lwoffii \\
\hline $\begin{array}{l}\text { type I secretion outer membrane } \\
\text { protein, TolC family }\end{array}$ & YDYLINVLH & Acinetobacter johnsonii SH046 \\
\hline $\begin{array}{l}14 \text {-alpha-glucan branching } \\
\text { enzyme GlgB }\end{array}$ & YEYMINVI & Clostridium sp. CAG:964 \\
\hline $\begin{array}{l}\text { ATP-dependent helicase/ } \\
\text { deoxyribonuclease subunit B/ } \\
\text { nuclease subunit B }\end{array}$ & YEFLID-IHAFE & Clostridium butyricum \\
\hline hypothetical protein & EYLVNVIH & Spiroplasma melliferum \\
\hline hypothetical protein & EY-IDVIHAKQYV & Paenibacillus pini \\
\hline (2Fe-2S)-binding protein & EYLVNITHAF & Pseudomonas fluorescens \\
\hline $\begin{array}{l}\text { putative } \mathrm{N} \text {-deacylase involved in } \\
\text { arginine and ornithine utilization }\end{array}$ & QYLLDVIHSFPY & Bacillus vireti \\
\hline hypothetical protein & NVVHDFEYVI & Vibrio sinaloensis \\
\hline $\begin{array}{l}\text { hypothetical protein } \\
\text { BN890_49820 }\end{array}$ & YAYLINELSGTVI-AFEY & Bacteroides xylanisolvens \\
\hline $\begin{array}{l}\text { 6-phosphogluconolactonase, } \\
\text { partial }\end{array}$ & YAYLINELSGTVI-AFEY & Bacteroides fragilis \\
\hline hypothetical protein & YEYLIYVDRIHA & Bacteroides \\
\hline hypothetical protein & LIDVIHAIHFII & Acinetobacter beijerinckii \\
\hline tRNA pseudouridine synthase A & YEYLIN-TRAFNPAQY & Mycoplasma pneumonia \\
\hline hypothetical protein & YEYLINV & Lachnospiraceae bacterium \\
\hline $\begin{array}{l}\text { binding--dependent transport } \\
\text { system inner membrane } \\
\text { component family protein }\end{array}$ & ISVIHAFQAFDMVYV & Mycobacterium avium subsp. avium 2285 \\
\hline sugar $\mathrm{ABC}$ transporter permease & ISVIHAFQAFDMVYV & Mycobacterium colombiense CECT 3035 \\
\hline $\begin{array}{l}\text { MULTISPECIES: ABC transporter } \\
\text { permease }\end{array}$ & ISVIHAFQAFDMVYV & Mycobacterium avium complex (MAC) \\
\hline hypothetical protein & YEYLIYVDRIHA & Bacteroides sp. CAG:661 \\
\hline $\begin{array}{l}\text { cytochrome oxidase assembly } \\
\text { protein }\end{array}$ & EYFINVIHLF & Leptospira \\
\hline $\mathrm{ABC}$ transporter permease & ISVIHAFQAFDMVYV & $\begin{array}{l}\text { Mycobacterium tuberculosis/colombiense/ intracellulare/ } \\
\text { avium complex }\end{array}$ \\
\hline 6-phosphogluconolactonase & YAYLINELSGTVI-AFEY & Bacteroides sp. D2 \\
\hline
\end{tabular}


Zamanzadeh Z. et al.

Table 3. Continued

\begin{tabular}{|c|c|c|}
\hline $\begin{array}{l}\text { aromatic amino acid } \\
\text { aminotransferase }\end{array}$ & EYLIKMMKKIH-QYVI & Bacterium LF-3 \\
\hline ac70-like protein & YNYLKNVIHA & Clanis bilineata nucleopolyhedro virus \\
\hline unnamed protein product & EYLINVI & Pseudomonas phage $O B P$ \\
\hline hypothetical protein SP126_00040 & EYVRNVAHAFDNVI & Salmonella phage FSL SP-126 \\
\hline putative membrane protein & LIKVIHGLEYII & Enterobacteria phage \\
\hline hypothetical protein SPC35_0038 & LIKVIHGLEYII & Salmonella phage Spc35 \\
\hline Flavodoxin & YL----HAFEYVI & Bacillus phage \\
\hline multifunctional replicase, partial & FEYLLNVFHA & Chara australis virus \\
\hline ORF140 & YEYEVKVVQLFQYI & Cydia pomonella granulovirus \\
\hline m152 protein & YEYLMNVFKAGRPIIFEY & Murid herpesvirus 1 \\
\hline hypothetical protein BpV1_188c & YEYLLN-IKTYQY & Bathycoccus \\
\hline Endonuclease & YEYLISNIH & Enterobacteria phage HK629 \\
\hline Protein MGF 505-7R & EFLINIIH , DYLI & African swine fever virus Malawi LIL 20/1 \\
\hline thymidylate kinase & YEYLIN & Halovirus HVTV-1 \\
\hline regulatory protein & IDVIHAIEYI & Escherichia phage $1720 a-02$ \\
\hline RNA polymerase subunit RPO147 & YEYLIN & Deerpox virus \\
\hline spike glycoprotein & $\begin{array}{l}\text { YEYFNN-IHAF } \\
\text { EYLI }\end{array}$ & Canine coronavirus \\
\hline spike protein, partial & YEYFNN-IHAF & Alphacoronavirus 1 \\
\hline envelope glycoprotein & LI-VIHALQY & Human immunodeficiency virus 1 \\
\hline putative ankyrin repeat protein & $\begin{array}{l}\text { LINVEQNI-FEYVI } \\
\text { FEYLI }\end{array}$ & Acanthamoeba polyphaga mimivirus \\
\hline $\begin{array}{l}\text { putative NAD }+ \text { dependent DNA } \\
\text { ligase }\end{array}$ & YEYIINILNA & Amsacta moorei entomopoxvirus \\
\hline DNA primase & ECLINKRSRDVIHAIFQ & uncultured Mediterranean phage uvMED \\
\hline Spike glycoprotein & YEYFNN-IHAF & Feline coronavirus \\
\hline spike protein/ S protein & YEYFNN-IHAF & Canine coronavirus \\
\hline $\mathrm{CI}$ repressor & IDVIHAIEY & Shigella phage \\
\hline regulatory protein & IDVIHAIEY & Salmonella phage \\
\hline
\end{tabular}

immune system with the PLP may have originated from a bacterial or viral infection and therefore, genetic makeup (the presence of the susceptible alleles in one person) and exposure to foreign organism contribute to the increasing risk of auto-immune diseases like MS.

\section{Discussion}

The causes of MS are unknown, but the most probable theory is suggested to be through a molecular mimicry process via infections that activation $T$ cells. $T$ cell reactivity to brain auto-antigens, particularly myelin antigens, plays a crucial role for commencing of the $\operatorname{MS}(5,6)$.

Molecular mimicry is also known as "crossreactivity" between self-antigens and immunodominant epitopes of the pathogens. Cross-reactivity can be the cause of many autoimmune disorders such as myasthenia gravis which occurs due to detectable antibodies produced against the human acetylcholine receptor. Cross-reactivity of the self-epitope with the antibodies produced against herpes simplex virus glycoprotein D suggests that the virus is associated with the initiation of myasthenia gravis (33).

Schloot et al. in 2001 have shown that isolated $\mathrm{T}$ cells from type 1 diabetes patients which are reactive to glutamic acid decarboxylase65 (GAD65) cross-react with the Coxsackie virus protein $\mathrm{P} 2 \mathrm{C}$ and pro-insulin protein (34).

There is a significant association between parvovirus B19 cross-reactivity and several auto-immune disorders including rheumatoid arthritis, systemic lupus, antiphospholipid syndrome and systemic sclerosis (35). Poole et al. in 2006 have demonstrated that antibodies produced against Epstein-Barr virus nuclear antigen-1 (EBNA-1) cross-react with lupus-associated autoantigens (36).

Therefore, molecular mimicry between the host and microbial proteins seems to be the main mechanism 
involved in the induction of autoimmunity and MS. There are numbers of evidence which prove the role of molecular mimicry in the mechanism of MS incidence. Due to the abundance of PLP in CNS myelin and its restriction to the CNS, it has been the most important candidate in the pathogenesis of MS.

The only known structure in the PDB was a monomeric peptide (KLIETYFSK) in complex with HLA-A0301 (PDB ID 2XPG), therefore, we modeled the protein.

We have taken a systematic approach based on available bioinformatic tools and three fragments were selected as the immunogenic epitopes. Whitham et al. (1991) have already shown that residue 43-64 of PLP could induce EAE in $\mathrm{PL} / \mathrm{J}$ mice. Other researchers have shown PLP peptides 139-151, 178-191, 104117 , and 57-70 are encephalitogenic for $\mathrm{SJL} / \mathrm{J}$ mice (22-25). In 1993 Pelfrey et al. have suggested that $\mathrm{T}$ cells can respond to the PLP 40-60 fragment. Greer et al. (2004) synthesized the fragments 41-58, 95-114, 178-191, 184-199, 190-209, 100-119, and 139-151 and treated MS patients' PBMC with such synthetic peptides. Although the result was the proliferation in response to the peptide fragments 184-199 and 290209 (29), they synthesized the fragments randomly without considering continuous epitopes processing ways in proteasome system of antigen presenting. Their prediction methods for recognition of the effective PLP epitopes in MS were based on the following criteria: Reports of encephalitogenic potential in animal models and preferential reactivity by MS T-cells.

The immune system has an unavoidable role in the pathogenesis of MS through the major histocompatibility complex (MHC) class II (15). Moreover, Continuous fragments which can be recognized by $\mathrm{T}$ cells and were processed by proteasome complex of antigen presenting cells are presented by MHCII. Besides, it was proposed that MHC class I has an important role in the initiation of MS too, because (i) CD8+ T cells are the most common cells in the lesions, (ii) MHC I molecule is up-regulated in the induced EAE mice using TMEV $(37,38)$.

Some MHC I and II alleles have been associated with MS and each class contributes to the risk of the disease, such as HLA-A24, HLA-DRB1*15, HLA-DRB1*04, HLADRB1*03:01, HLADRB2, HLADQA1*01:02/DQB1*0602, HLADQA1*04:01/ DQB $1 * 0402, \quad$ HLADQA $1 * 05: 01 /$ DQB1*0201, and HLADQA1*05:01/DQB1*0301. While it was shown that HLA-A2 has a protective effect against MS (29, $39,40)$. Selection of the different HLAs involved in the MS initiation has improved the prediction validity in order to use results at the population scale.

As mentioned above, previous studies have experimentally investigated the immunogenic epitopes of the PLP by (i) induction of EAE with overlapping peptides or (ii) their effects on patients' $\mathrm{T}$ cells proliferation $(29,30)$. Also, some reports have focused on homology between brain auto-antigens and microorganisms which may have a role in MS initiation through molecular mimicry mechanism (9). Nowadays it is possible to predict immunogenic epitopes in-silico without a need for the synthesis of each fragment and test them. Such systematic approaches are mainly needed for the prediction of the immunogenic epitopes which have high homology with the microorganisms.

As indicated above, in the initiation phase of this research, the so-called "in-silico Phase", was done through comprehensive predictions by bioinformatics tools despite other published articles. PLPs antigenic epitopes sequences were predicted as the myelinspecific protein with common antigenicity among the PLPs and a wide range of membrane protein as pathogen epitopes.

On the other hand, several useful bioinformatics software has been developed to predict the most probable epitopes. In this study, the best encephalitogenic epitopes were predicted according to the bioinformatics tools that may provide reliable results for researches. In this research, fragments with the best scores were selected, so they were aligned together in order to choose the most overlapping regions.

BLAST results for different predicted peptide epitopes have revealed high similarity $(>50 \%$ in most cases) between human PLP to a number of bacterial and viral components. Most of the BLAST hit results were related to the membrane proteins (such as; Transporters), permeases, transferases (Histidine kinase), spike protein etc. This shows the epitopes of PLP may induce MS through a molecular mimicry mechanism.

As it is seen in Table 3 (BLASTP results), the candidate epitope showed a high similarity with several antigenic bacterial and viral membrane proteins such as spike protein. These findings can provide evidence for the role of molecular mimicry in the initiation of MS. Based on this theory and the results of this research, the cross-reaction between PLPs and bacterial or viral epitopes can ignite a cascade of immune responses which could lead to MS.

Based on the results of this work PLP $_{58-74}$ could be a candidate as an immunodominant epitope of the PLP. We tested our hypothesis experimentally, therefore, patients and healthy individuals' peripheral blood mononuclear cells (PBMCs) were treated by 
$\mathrm{PLP}_{58-74}$ and its proliferative effects were evaluated through proliferating cell nuclear antigen (PCNA) gene expression changes assessment by real time PCR and immunocytochemistry assay. Experimental results showed that PLP $_{58-74}$ could induce proliferation in patient's PBMCs while it did not influence the PBMCs of the healthy individuals (41)

Massilamany et al. (2010) showed that there is a cross reaction between PLP $_{139-151}$ and Acanthamoeba castellanii (ACA) ${ }_{83-95}$ and both of them induce EAE in $\mathrm{SJL} / \mathrm{J}$ mice. They observed that both epitopes (PLP ${ }_{139}$ ${ }_{151}$ and $\mathrm{ACA}_{83-95}$ ) could stimulate proliferation of the T cell derived from lymph nodes (7). Wegmann et al. (2014) observed that EAE mice induced using PLP 139-151 $_{15}$ could be treated by modified $\mathrm{ACA}_{83-95}$. They stated that $\mathrm{ACA}_{83-95}$ prevents the development of rEAE (42). Also, in 2013 Badawi and Siahan showed that multivalent bifunctional peptide inhibitors $\left(\mathrm{MVB}_{\mathrm{MOG} / \mathrm{PLP}}\right)$ can suppress MOG38-50- and PLP139-151-induced EAE (43).

Olson et al. (2001) engineered a nonpathogenic TMEV variant to encode the PLP $_{139-151}$ epitope for the assessment of molecular mimicry potential in the initiation of MS. They showed that there is a cross reaction between pathogenic TMEV, PLP139-151 epitope, and Haemophilus influenza which could induce $\mathrm{EAE}$ in SJL/J mice (44).

Many microorganisms have been suspected of involved in MS. Greene, (2008) has indicated that Mycobacterium avium and Haemophilus influenza can induce EAE in $\mathrm{dTgH} 2 \mathrm{Aneg}$ mice (45). Also, Westall (2006) has stated that gut bacteria such as mycobacterium, clostridium, salmonella etc., might be associated with MS initiation (46).

Among the main achievements of the present study was the modeling of PLP. As there wasn't any tertiary structure of this protein available in the Protein Data Bank (PDB), for characterizing the structural properties of this protein we modeled this protein.

The results of this study contribute to a better understanding and clarification of the mechanism of the disease and causes of MS. These results can be used to prevent the initiation of MS, immunotherapy, and individual medicine in future.

\section{Conclusion}

Herein, we tried to find immunodominant epitopes for cytotoxic and helper $T$ cells based on susceptible alleles to MS, checking their matches in microbial components and to clarify some of the structural characteristics of the PLP as an important protein in MS pathogenesis. By reliable and accurate prediction of the consensus epitopes, it is not necessary to synthesize all PLP fragments and examine their immunogenicity experimentally (in vitro). Therefore, researchers can achieve better results in a shorter time and at a lower cost. Besides, they could check if the consensus predicted epitopes have a cross reaction with the foreign particles, immunotherapeutic regimens, and gain a better understanding of the mechanism of MS diseases. This approach allows the development of peptide-based pharmacotherapy for MS and promises for personalized medicine in future.

\section{Acknowledgments}

This work was supported by a grant from the National Institute of Genetic Engineering and Biotechnology (NIGEB) of Iran.

\section{References}

1. Wucherpfennig KW, Weiner HL, Hafler DA. T-cell recognition of myelin basic protein. Immunol. today. 1991;12(8):277-82. doi:10.1016/0167-5699(91)90126-E

2. Kondo T, Yamamura T, Inobe J-i, Ohashi T, Takahashi K, Tabira T. TCR repertoire to proteolipid protein (PLP) in multiple sclerosis (MS): homologies between PLP-specific T cells and MS-associated T cells in TCR junctional sequences. Int. Immunol. 1996;8(1):123-30. doi: 10.1093/intimm/8.1.123

3. McKay KA, Kwan V, Duggan T, Tremlett H. Risk Factors Associated with the Onset of Relapsing-Remitting and Primary Progressive Multiple Sclerosis: A Systematic Review. BioMed Res. Int. 2015;2015. doi.org/10.1155/2015/817238

4. Goldenberg MM. Pharmaceutical approval update. J Clin. Pharm.Ther. 2009;34(10):569.

5. Elong-Ngono A, Pettré S, Salou M, Bahbouhi B, Soulillou $\mathrm{J}-\mathrm{P}$, Brouard S, et al. Frequency of circulating autoreactive $\mathrm{T}$ cells committed to myelin determinants in relapsing-remitting multiple sclerosis patients. Clin. Immunol. 2012;144(2):117-26. doi: 10.1016/j.clim.2012.05.009. 6 .

6. Olson JK, Croxford JL, Calenoff MA, Dal Canto MC, Miller SD. A virus-induced molecular mimicry model of multiple sclerosis. J Clin. Invest. 2001;108(2):311-8. doi: 10.1007/3540-30791-5_3

7. Massilamany C, Steffen D, Reddy J. An epitope from $<$ i $>$ Acanthamoeba castellanii $</ \mathrm{i}>$ that cross-react with proteolipid protein 139-151-reactive $\mathrm{T}$ cells induces autoimmune encephalomyelitis in SJL mice. $J$ Neuroimmunol. 2010;219(1):17-24. doi: http://dx.doi.org/10.1016/j. jneuroim.20099.11.006

8. Toohey L. Impact of Dietary Antigens on Multiple Sclerosis. J. Nutr. Environ. Med. 2004;14(4):31926. doi: $10.1080 / 13590840500088446$. doi: $10.1080 / 13590840500088446$

9. Wucherpfennig KW, Strominger JL. Molecular mimicry in T cell-mediated autoimmunity: viral peptides activate human T cell clones specific for myelin basic protein. Cell. 1995;80(5):695705. DOI: 10.1016/0092-8674(95)90348-8

10. Marín N, Eixarch H, Mansilla M, Rodríguez-Martín E, Mecha M, Guaza C, et al. Anti-myelin antibodies play an important 
role in the susceptibility to develop proteolipid protein-induced experimental autoimmune encephalomyelitis. Clin. Exper. Immunol 2014;175(2):202-7. doi: 10.1111/cei.12233

11. Greer JM, Pender MP. Myelin proteolipid protein: an effective autoantigen and target of autoimmunity in multiple sclerosis. $J$. Autoimmun. 2008;31(3):281-7.

12. Bachstetter AD, Webster SJ, Van Eldik LJ, Cambi F. Clinically relevant intronic splicing enhancer mutation in myelin proteolipid protein leads to progressive microglia and astrocyte activation in white and gray matter regions of the brain. $J$ Neuroinflammation. 2013;10:146. doi: 10.1186/1742-2094-10146

13. McLaughlin M, Hunter D, Thomson C, Yool D, Kirkham D, Freer A, et al. Evidence for possible interactions between PLP and DM20 within the myelin sheath. Glia. 2002;39(1):31-6. doi: 10.1002/glia.10091

14. Macklin WB. Marjorie Lees (1923-2012). J Neurochem. 2012;123(5):895-6. DOI: 10.1111/jnc. 12033

15. Mecha M, Carrillo-Salinas FJ, Mestre L, Feliú A, Guaza C. Viral models of multiple sclerosis: Neurodegeneration and demyelination in mice infected with Theilers virus. Prog Neurobiol. 2012. DOI:10.1016/j.pneurobio.2012.11.003

16. Ng DP, Deber CM. Modulation of the oligomerization of myelin proteolipid protein by transmembrane helix interaction motifs. Biochemistry. 2010;49(32):6896-902. DOI: 10.1021/bi100739r

17. Yamaguchi Y, Ikenaka K, Niinobe M, Yamada H, Mikoshiba K. Myelin proteolipid protein (PLP), but not DM-20, is an inositol hexakisphosphate-binding protein. Journal of Biological Chemistry. 1996;271(44):27838-46.

18. Popot J-L, Dinh DP, Dautigny A. Major myelin proteolipid: the

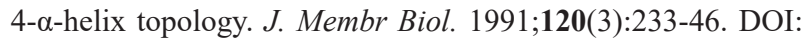
10.1007/BF01868534

19. Pereira GB, Meng F, Kockara NT, Yang B, Wight PA. Targeted deletion of the antisilencer/enhancer (ASE) element from intron 1 of the myelin proteolipid protein gene (Plp1) in mouse reveals that the element is dispensable for Plp1 expression in brain during development and remyelination. Journal of neurochemistry. 2013;124(4):454-65. DOI: 10.1111/jnc.12092

20. Göbel K, Bittner S, Ruck T, Budde T, Wischmeyer E, Döring F, et al. Active immunization with proteolipid protein (190209) induces ascending paralysing experimental autoimmune encephalomyelitis in $\mathrm{C} 3 \mathrm{H} / \mathrm{HeJ}$ mice. Journal of immunological methods. 2011;367(1):27-32. DOI:10.1016/j.jim.2010.12.018

21. Whitham R, Jones R, Hashim G, Hoy C, Wang R, Vandenbark A, et al. Location of a new encephalitogenic epitope (residues 43 to 64) in proteolipid protein that induces relapsing experimental autoimmune encephalomyelitis in PL/J and (SJL x PL) F1 mice. The Journal of Immunology. 1991;147(11):3803-8.

22. Tuohy V, Lu Z, Sobel R, Laursen R, Lees M. Identification of an encephalitogenic determinant of myelin proteolipid protein for SJL mice. The Journal of Immunology. 1989;142(5):1523-7.

23. Greer JM, Kuchroo VK, Sobel RA, Lees M. Identification and characterization of a second encephalitogenic determinant of myelin proteolipid protein (residues 178-191) for SJL mice. The Journal of Immunology. 1992;149(3):783-8.

24. Tuohy VK, Thomas DM. Sequence 104-117 of myelin proteolipid protein is a cryptic encephalitogenic $\mathrm{T}$ cell determinant for SJL/J mice. Journal of neuroimmunology. 1995;56(2):161-70. DOI: 10.1016/0165-5728(94)00143-C

25. Greer JM, Sobel RA, Sette A, Southwood S, Lees MB, Kuchroo VK. Immunogenic and encephalitogenic epitope clusters of myelin proteolipid protein. The Journal of Immunology. 1996;156(1):371-9.

26. Pelfrey CM, Trotter JL, Tranquill LR, McFarland HF. Identification of a novel $\mathrm{T}$ cell epitope of human proteolipid protein (residues 40-60) recognized by proliferative and cytolytic $\mathrm{CD} 4<\sup >+</$ sup $>\mathrm{T}$ cells from multiple sclerosis patients. Journal of neuroimmunology. 1993;46(1):33-42. DOI: 10.1016/0165-5728(93)90231-M

27. Pelfrey CM, Trotter JL, Tranquill LR, McFarland HF. Identification of a second $\mathrm{T}$ cell epitore of human proteolipid protein (residues 89-106) recognized by proliferative and cytolytic $\mathrm{CD} 4<\sup >+</$ sup $>\mathrm{T}$ cells from multiple sclerosis patients. Journal of neuroimmunology. 1994;53(2):153-61. DOI: 10.1016/0165-5728(94)90025-6

28. Markovic-Plese S, Fukaura H, Zhang J, Al-Sabbagh A, Southwood S, Sette A, et al. T cell recognition of immunodominant and cryptic proteolipid protein epitopes in humans. The Journal of Immunology. 1995;155(2):982-92. DOI: $10.1016 / 0165-5728(94) 90423-5$

29. Greer JM, Csurhes PA, Cameron KD, McCombe PA, Good MF, Pender MP. Increased immunoreactivity to two overlapping peptides of myelin proteolipid protein in multiple sclerosis. Brain. 1997;120(8):1447-60. DOI: DOI:10.1093/ brain/120.8.1447

30. Kaushansky N, Altmann DM, David CS, Lassmann H, Ben-Nun A. DQB1* 0602 rather than DRB1* 1501 confers susceptibility to multiple sclerosis-like disease induced by proteolipid protein (PLP). J Neuroinflammation. 2012;9:29. DOI: 10.1186/17422094-9-29

31. Lovell SC, Davis IW, Arendall WB, de Bakker PI, Word JM, Prisant MG, et al. Structure validation by $\mathrm{C} \alpha$ geometry: $\phi, \psi$ and $\mathrm{C} \beta$ deviation. Proteins: Structure, Function, and Bioinformatics. 2003;50(3):437-50. DOI: 10.1002/prot.10286

32. WU XD, Shang B, Yang RF, Hao Y, Hai Z, Xu S, et al. The spike protein of severe acute respiratory syndrome (SARS) is cleaved in virus infected Vero-E6 cells. Cell research. 2004;14(5):400-6.

33. Schwimbeck P, Dyrberg T, Drachman D, Oldstone M. Molecular mimicry and myasthenia gravis. J Clin Invest. 1989;840:117482. doi:10.1038/sj.cr.7290240

34. Schloot N, Willemen S, Duinkerken G, Drijfhout J, De Vries R, Roep B. Molecular mimicry in type 1 diabetes mellitus revisited: T-cell clones to GAD65 peptides with sequence homology to Coxsackie or proinsulin peptides do not crossreact with homologous counterpart. Human immunology. 2001;62(4):299309. DOI:10.1016/S0198-8859(01)00223-3

35. Lunardi C, Tinazzi E, Bason C, Dolcino M, Corrocher R, Puccetti A. Human parvovirus B19 infection and autoimmunity. Autoimmunity reviews. 2008;8(2):116-20.

36. Poole BD, Scofield RH, Harley JB, James JA. EpsteinBarr virus and molecular mimicry in systemic lupus erythematosus. Autoimmunity. 2006;39(1):63-70. DOI:10.1080/08916930500484849

37. Tsunoda I, Fujinami RS. Neuropathogenesis of Theiler's murine encephalomyelitis virus infection, an animal model for multiple sclerosis. Journal of Neuroimmune Pharmacology. 2010;5(3):355-69.

38. Fletcher J, Lalor S, Sweeney C, Tubridy N, Mills K. T cells in multiple sclerosis and experimental autoimmune encephalomyelitis. Clinical \& Experimental Immunology. 2010;162(1):1-11. DOI: 10.1111/j.1365-2249.2010.04143.x

39. McMahon RM, Friis L, Siebold C, Friese MA, Fugger L, Jones 
EY. Structure of HLA-A* 0301 in complex with a peptide of proteolipid protein: insights into the role of HLA-A alleles in susceptibility to multiple sclerosis. Acta Crystallographica Section D: Biological Crystallography. 2011;67(5):447-54. DOI:10.1107/S0907444911007888

40. Grau-López L, Raïch D, Ramo-Tello C, Naranjo-Gómez M, Dàvalos A, Pujol-Borrell R, et al. Myelin peptides in multiple sclerosis. Autoimmunity reviews. 2009;8(8):650-3.

41. Zamanzadeh Z, Ahangari Gh, Ataie M, Pouragahi S, Nabavi SM, et al. Myelin Proteolipid Protein (58-74), Could it be as a candidate of a Putative Epitope Associated with Pathogenesis of Multiple Sclerosis? Iranian journal of Allergy and Asthma and Immunology. 2016 (Zamanzadeh et al. in press)

42. Wegmann KW, Bouwer HA, Whitham RH, Hinrichs DJ. Eluding anaphylaxis allows peptide-specific prevention of the relapsing stage of experimental autoimmune encephalomyelitis. Journal of neuroimmunology. 2014;274(1):46-52. DOI:10.1016/j. jneuroim.2014.06.011

43. Badawi AH, Siahaan TJ. Suppression of MOG-and PLPinduced experimental autoimmune encephalomyelitis using a novel multivalent bifunctional peptide inhibitor. Journal of neuroimmunology. 2013;263(1):20-7. DOI: 10.1016/j. jneuroim.2013.07.009

44. Olson JK, Croxford JL, Calenoff MA, Dal Canto MC, Miller SD. A virus-induced molecular mimicry model of multiple sclerosis. Journal of Clinical Investigation. 2001;108(2):311.

45. Greene MT, Ercolini AM, DeGutes M, Miller SD. Differential induction of experimental autoimmune encephalomyelitis by myelin basic protein molecular mimics in mice humanized for HLA-DR2 and an MBP 85-99-specific T cell receptor. Journal of autoimmunity. 2008;31(4):399-407.

46. Westall FC. Molecular mimicry revisited: gut bacteria and multiple sclerosis. Journal of clinical microbiology. 2006;44(6):2099-104 